\title{
On Achieving the Shannon Bound in Cellular Systems
}

\author{
Markus RUPP, Christian MEHLFÜHRER, and Sebastian CABAN \\ Institute of Communications and Radio-Frequency Engineering, Vienna University of Technology, Vienna, Austria \\ $\{$ mrupp, chmehl, scaban\}@nt.tuwien.ac.at
}

\begin{abstract}
This contribution provides insight into the performance of the currently deployed $3 G$ cellular systems HSDPA and WiMAX. In extensive measurement campaigns we measured the physical layer throughput of these $3 G$ systems in different environments (alpine and urban) and compare the results rigorously to their upper bounds derived from the well known Shannon capacity. By separating the observed losses into a channel state information loss, a design loss, and an implementation loss, we gain more insight into the performance of the different standards which in turn allows us to compare them better and to localize their shortcomings. In general, we find that implementations of the current standards still operate about $10 \mathrm{~dB}$ away from the Shannon bound.
\end{abstract}

\section{Keywords}

MIMO, HSDPA, WiMAX, performance measures, testbed.

\section{Introduction}

We implemented, measured, and studied the following two standardized cellular systems:

- The WiMAX physical layer as defined in IEEE 802.16-2004 [1] was developed to provide wireless internet access for stationary and low-mobility users. In the standard, three different physical layers for WiMAX are defined, namely the Single Carrier (SC) [1, Sections 8.1 and 8.2], the Orthogonal Frequency Division Multiplexing (OFDM) [1, Section 8.3], and the Orthogonal Frequency Division Multiple Access (OFDMA) [1, Section 8.4] physical layers. The SC physical layer is designed for directional radio links with Line-Of-Sight (LOS), whereas the OFDM and OFDMA physical layers are designed for Non-LineOf-Sight (NLOS) conditions. The OFDM physical layer utilizes 256 narrow-band sub-carriers to modulate the data symbols to be transmitted. Multiple users are supported by Time-Division Multiple Access (TDMA). The OFDMA physical layer, in contrast, provides in addition to TDMA also multiple access by assigning specific sub-carrier subsets to individual users [2]. In this work, we only consider the OFDM physical layer of WiMAX.
- High Speed Downlink Packet Access (HSDPA) [3] was introduced in Release 5 of the Universal Mobile Telecommunication System (UMTS) to provide high data rates to mobile users [4-7]. This is achieved by several techniques like fast link adaptation [8], fast hybrid automated repeat request [9], and fast scheduling [10]. In contrast to the pure transmit power adaptation performed in UMTS, fast link adaptation in HSDPA adjusts the data rate and the number of spreading codes depending on a so-called Channel Quality Indicator (CQI) feedback. MIMO HSDPA [11], recently standardized in Release 7 of UMTS, further increases the maximum downlink data rate by spatially multiplexing two independently coded and modulated data streams. Additionally, channel-adaptive spatial precoding is implemented at the basestation. The standard defines a set of precoding vectors and one of them is chosen based on a so-called Precoding Control Indicator (PCI) feedback obtained from the user equipment.

This paper is organized as follows: In Section II we define upper bounds for the measured throughput and identify three different throughput losses. Section III presents measurement results and analyzes the different losses. Finally, Section IV provides a conclusion explaining the reasons for the different losses in HSDPA and WiMAX.

\section{Performance Metrics}

Given the channel impulses $h_{k}^{\left(n_{r}, n_{t}\right)}$ of an $N_{\mathrm{T}} \times N_{\mathrm{R}}$ MIMO system (for example by measuring them at high SNR) one can compute the so called Shannon capacity

$$
C(\mathrm{SNR})=\max _{\operatorname{tr}\{\mathbf{R}\} \leq 1} \log _{2} \operatorname{det}\left(\mathbf{I}+\frac{\mathrm{SNR}}{N_{\mathrm{T}}} \mathbf{H R H} \mathbf{H}^{\mathrm{H}}\right)
$$

where each entry of $\mathbf{H}$ is given by $h_{k}^{\left(n_{r}, n_{t}\right)} ; 1 \leq n_{r} \leq$ $N_{\mathrm{R}}, 1 \leq n_{t} \leq N_{\mathrm{T}}$ and $\mathbf{R}$ is the optimal precoding matrix obtained by waterfilling. This measure tells us how much $\mathrm{bit} / \mathrm{s} / \mathrm{Hz}$ we are able to transmit at best. As the calculation of the optimal precoding matrix $\mathbf{R}$ requires precise information about the channel $\mathbf{H}$, typically obtained by feedback from receiver to transmitter, it is of interest to assess the so-called mutual information that only requires to know the 
SNR:

$$
I(\mathrm{SNR})=\log _{2} \operatorname{det}\left(\mathbf{I}+\frac{\mathrm{SNR}}{N_{\mathrm{T}}} \mathbf{H H}^{\mathrm{H}}\right) .
$$

Because capacity and mutual information only differ in the knowledge of the channel state information at the transmitter, we define their difference as the channel state information loss

$$
L_{\mathrm{CSI}}\left(P_{\mathrm{Tx}}\right) \triangleq C\left(P_{\mathrm{Tx}}\right)-I\left(P_{\mathrm{Tx}}\right) .
$$

Note that rather than plotting $L_{\mathrm{CSI}}$ as a function of SNR we have changed the notation to transmit power $P_{\mathrm{Tx}}$. There are several reasons for this: firstly, we measure over transmit power, secondly, the mean received power in MIMO scenarios is significantly different at each antenna, and thirdly, when employing adaptive precoding at the transmitter, the transmit power remains unchanged while the receive SNR varies.

Given a certain wireless standard, we now have to take an overhead into account.

- In HSDPA this overhead is given by the amount of power devoted to the pilots and synchronization sequences. This kind of overhead affects the mutual information by shifting the SNR to lower values. Also the roll-off factor of 0.22 , limiting the useful bandwidth, needs to be taken into account. All these factors lower the mutual information.

- In OFDM systems such as WiMAX and LTE, pilots consume bandwidth and the cyclic prefix consumes transmission time. Thus, these losses are proportional to the mutual information and not to the SNR.

Taking all mentioned losses into account, we obtain the so called achievable mutual information [12-14]

$$
I_{\mathrm{a}}(\mathrm{SNR})=\beta \log _{2} \operatorname{det}\left(\mathbf{I}+\frac{\alpha \mathrm{SNR}}{N_{\mathrm{T}}} \mathbf{H R}_{\mathrm{q}} \mathbf{H}^{\mathrm{H}}\right),
$$

with $\alpha \leq 1$ and $\beta \leq 1$. In case of precoding (MIMO HSDPA), the matrix $\mathbf{R}_{\mathrm{q}}$ accounts for the quantization of the precoding matrices. The specific values of $\alpha$ and $\beta$ depend on the detailed specification of the wireless transmission system. In case of WiMAX transmissions, we obtain $\alpha=1$ and $\beta=0.56$, if the parameters are set as in [15]. In case of HSDPA transmissions, we obtain $\alpha=0.4$ (if $-4 \mathrm{~dB}$ of the total transmit power is assigned to the data channels) and $\beta=0.82$ (corresponding to the inverse of the HSDPA root-raised cosine roll-off factor of 1.22).

It is worthwhile noting that all precoding matrices in HSDPA for $2 \times 2$ MIMO transmission are unitary $\left(\mathbf{R}_{\mathrm{q}}\right.$ is always equal to an identity matrix), thus not altering the achievable mutual information $I_{\mathrm{a}}$ at all. In case of $2 \times 2$ MIMO WiMAX, however, Alamouti space-time coding [16] is employed at the transmitter. Thus, by inserting the virtual MIMO channel matrix of the Alamouti space-time code

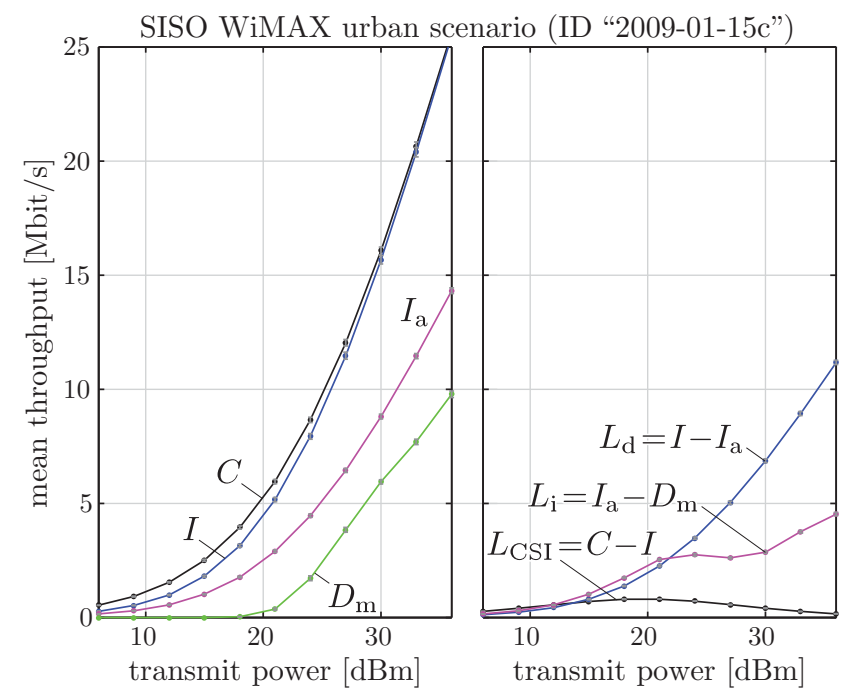

Fig. 1. Throughput and throughput losses of the SISO WiMAX system in the urban environment, obtained from 484 small scale fading channel realizations.

and setting $\mathbf{R}_{\mathrm{q}}=\mathbf{I}$ in Equation (4), we obtain the achievable mutual information for MIMO WiMAX. Since Alamouti space-time coding is not capacity achieving when two receive antennas are utilized, the consideration of the spacetime coding lowers the achievable mutual information.

The achievable mutual information includes the losses purely caused by the design of the system, therefore we define the difference between mutual information and achievable mutual information as the design loss:

$$
L_{\mathrm{d}}\left(P_{\mathrm{Tx}}\right) \triangleq I\left(P_{\mathrm{Tx}}\right)-I_{\mathrm{a}}\left(P_{\mathrm{Tx}}\right),
$$

where we again switched the notation to transmit power $P_{\text {Tx }}$. Note that the design loss is completely independent of the quality of the implementation.

The achievable mutual information is an upper bound for the physical layer throughput. It can only be achieved, if the implementation of the transmission system is optimal, that is, no further losses occur. However, due to for example imperfect channel knowledge at the receiver or suboptimal receiver design (because of complexity issues), a so-called implementation loss $L_{\mathrm{i}}$ further limits the physical layer throughput. We define the difference between the measured (achieved) throughput $D_{\mathrm{m}}(\mathrm{SNR})$ and the achievable mutual information $I_{\mathrm{a}}$ as the implementation loss:

$$
L_{\mathrm{i}}\left(P_{\mathrm{Tx}}\right) \triangleq I_{\mathrm{a}}\left(P_{\mathrm{Tx}}\right)-D_{\mathrm{m}}\left(P_{\mathrm{Tx}}\right) .
$$

Note that according to our definition of the implementation loss, also other losses that are not purely caused by the implementation of the transmission system are included in the implementation loss. For example, transmission systems currently employed are not transmitting Gaussian signals of infinite length as it would be required to achieve the Shannon bound. Furthermore, at high transmit power levels (corresponding to high receive SNRs) the throughput of 
any wireless transmission system saturates because no larger adaptive modulation and coding schemes are available. This fact is currently not reflected in our definition of the achievable throughput definition and causes an increasing implementation loss at high SNR.

\section{Measurement Results}

As the paper size is quite limited, we cannot provide sufficient details on our measurement setup here. Instead we refer the interested reader to the references [17-21]. In short, the throughput is measured by transmitting and receiving the standard compliant signals. The bounds are calculated from the channel coefficients estimated from the received signals at highest $P_{\mathrm{Tx}}$.

In this paper, we exhibit results obtained in two different environments. The first one is an alpine environment in which the transmitter is placed in a distance of $5.7 \mathrm{~km}$ to the receiver. This environment is characterized by an essentially strong LOS component and moderate scattering with an RMS delay spread of $260 \mathrm{~ns}$. The second environment is an urban one with only $430 \mathrm{~m}$ distance between transmitter and receiver. In this environment the scattering is much richer with an RMS delay spread of $1.1 \mu$ s and no LOS component. In this work, we present only results for SISO and $2 \times 2$ MIMO transmissions with cross-polarized transmit antennas although many more situations were investigated.

\subsection{SISO Results}

In Figure 1 we plot on the left-hand side the capacity $C$, the mutual information $I$, the achievable mutual information $I_{\mathrm{a}}$, and the measured throughput $D_{\mathrm{m}}$. On the right-hand side we plot the channel state information loss $L_{\mathrm{CSI}}$, the design loss $L_{\mathrm{d}}$, and the implementation loss $L_{\mathrm{i}}$, as defined in Equations (3), (5), and (6). The left-hand side of Figure 1 shows that the total SNR loss of the measured throughput $D_{\mathrm{m}}$ compared to the capacity $C$ amounts to $9 \mathrm{~dB}$ at $5 \mathrm{Mbit} / \mathrm{s}$. This loss is further increasing with the SNR and reaches about $11 \mathrm{~dB}$ at $10 \mathrm{Mbit} / \mathrm{s}$.

Figure 2 shows the various losses of SISO WiMAX and SISO HDSPA transmissions in the alpine (left-hand side) and the urban environment (right-hand side). We find the following results for alpine as well as urban environments:

- The channel state information loss $L_{\mathrm{CSI}}$ is negligibly small compared to the other losses, as it has already been pointed out in [22].

- The design loss $L_{\mathrm{d}}$ is monotonically increasing with transmit power. Up to about $30 \mathrm{dBm}$, the values are very similar for WiMAX and HSDPA but for higher transmit power the design loss of HSDPA starts saturating while it keeps increasing for WiMAX.

- The implementation loss $L_{\mathrm{i}}$ is moderately small for
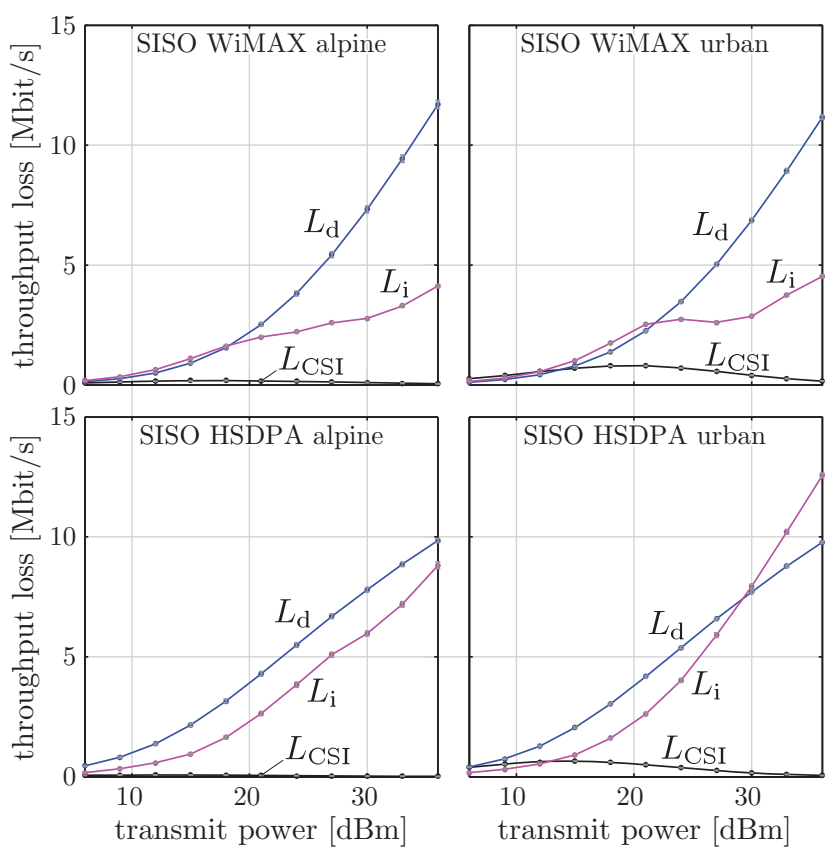

Fig. 2. Throughput losses of the SISO WiMAX and the SISO HSDPA systems in the alpine (ID "2008-09-23") and the urban environments (ID "2009-01-15c").
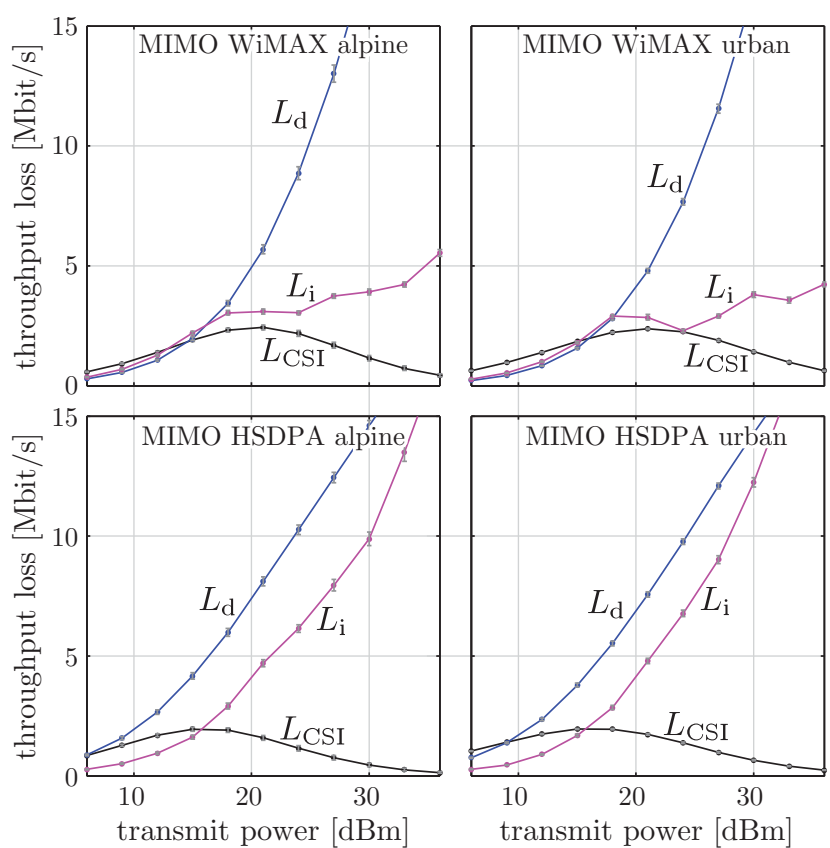

Fig. 3. Throughput losses of the MIMO WiMAX and the MIMO HSDPA systems in the alpine (ID "2008-09-23") and the urban environments (ID "2009-01-15c"). 
WiMAX and only slightly increasing with the transmit power while for HSDPA it is monotonously increasing.

The only relevant difference between alpine and urban environments we encounter in SISO HSDPA, where the implementation loss $L_{\mathrm{i}}$ at high SNR is significantly larger in the urban environment. The reason for this is the increased self interference (inter-chip interference) in the richer scattering with larger RMS delay spread. For WiMAX the large RMS delay spread has no influence since the OFDM transmission with cyclic prefix converts the frequency selective channel into several independent frequency flat subchannels.

\subsection{MIMO Results}

We repeated the measurements with a $2 \times 2$ MIMO system and display the results of the various losses of the WiMAX and HSDPA transmissions in Figure 3 for the alpine (left-hand side) and the urban environment (righthand side).

We find the following results for WiMAX and for HSDPA:

- The channel state information loss $L_{\mathrm{CSI}}$ has increased but is still the smallest compared to the other losses.

- The design loss $L_{\mathrm{d}}$ is again monotonically increasing with transmit power. At low transmit power, the design loss is larger in case of HSDPA transmission. At large transmit power it is the other way round.

- The implementation loss $L_{\mathrm{i}}$ shows a similar behavior as in the case of SISO transmissions

In contrast to the SISO transmissions, MIMO HSDPA shows no significant difference between alpine and urban environments.

\section{Conclusion}

In this work we presented measurement results for WiMAX and HSDPA in alpine and urban environments. We introduced three different throughput losses in order to analyze the performance. In particular, the introduced losses are:

1. The channel state information loss $L_{\mathrm{CSI}}$ (capacity minus mutual information): this typically small loss is given by the unknown channel state information at the transmitter. For SISO and cross polarized $2 \times 2$ MIMO systems this loss can be neglected compared to the other losses. Only in antenna arrays with at least four antennas this loss is worth considering [22]. In order to combat this loss, a significant amount of feedback information is required.
2. Design loss $L_{\mathrm{d}}$ (mutual information minus achievable mutual information): In case of WiMAX, this loss increases more dramatically with transmit power than in case of HSDPA.

3. Implementation loss $L_{\mathrm{i}}$ (achievable mutual information minus measured throughput): The implementation loss behaves very differently for WiMAX and HSDPA. In environments with large RMS delay spread (urban environment with $1.1 \mu \mathrm{s}$ ) the implementation loss of HSDPA is extremely large due to self interference while in small RMS delay spread areas (alpine environment with $260 \mathrm{~ns}$ ) the behavior is different. In WiMAX the different delay spread has no impact as the cyclic prefix was selected sufficiently large.

\section{Acknowledgement}

This work has been funded by the Christian Doppler Laboratory for Wireless Technologies for Sustainable Mobility, the Federal Ministry of Economy, Family and Youth and the National Foundation for Research, Technology and Development. The authors thank their industrial partners mobilkom austria AG and Kathrein-Werke KG as well as Christoph Mecklenbräuker.

\section{References}

[1] IEEE Standard for Local and Metropolitan Area Networks; Part 16: Air Interface for Fixed Broadband Wireless Access Systems, IEEE Std. 802.16-2004. [Online] 2004. Available at: http://standards.ieee.org/getieee802/download/802.16-2004.pdf

[2] GHOSH, A., WOLTER, D. R., ANDREWS, J. G., CHEN, R. Broadband wireless access with WiMax/802.16: current performance benchmarks and future potential. IEEE Communications Magazine, 2005, vol. 43, no. 2, p. $129-136$.

[3] Technical Specification Group Radio Access Network; Physical Layer Procedures (FDD) (3GPP TS 25.214 v7.7.0). 3GPP. [Online] 2007. Available at: http://www.3gpp.org/ftp/Specs/htmlinfo/25214.htm

[4] KOLDING, T. E., PEDERSEN, K. I., WIGARD, J., FREDERIKSEN, F., MOGENSEN, P. E. High speed downlink packet access: WCDMA evolution. IEEE Vehicular Technology Society News, 2003 , p. $4-10$.

[5] MOULSLEY, T. Throughput of high speed downlink packet access for UMTS. In Proc. $2^{\text {nd }}$ IEEE International Conference on $3 G$ Mobile Communication Technologies 2001 (3G 2001). 2001, p. 363 367.

[6] PARKVAll, S., DAhlMAN, E., FRENGER, P., BEMING, P., PERSSON, M. The evolution of WCDMA towards higher speed downlink packet data access. In Proc. 53 ${ }^{\text {rd }}$ IEEE Vehicular Technology Conference 2001 (VTC2001-Spring), vol. 3. 2001, p. 2287 2291.

[7] PARKVALl, S., DAHLMAN, E., FRENGER, P., BEMING, P., PERSSON, M. The high speed packet data evolution of WCDMA. In Proc. $12^{\text {th }}$ IEEE International Symposium on Personal, Indoor and Mobile Radio Communications 2001 (PIMRC 2001), vol. 2. 2001.

[8] NAKAMURA, M., AWAD, Y., VADGAMA, S. Adaptive control of link adaptation for high speed downlink packet access (HSDPA) in W-CDMA. In Proc. $5^{\text {th }}$ IEEE International Symposium on Wireless Personal Multimedia Communications 2002, vol. 2. 2002, p. 382 386. 
[9] DAS, A., KHAN, F., SAMPATH, A., SU, H.-J. Performance of hybrid ARQ for high speed downlink packet access in UMTS. In Proc. $54^{\text {th }}$ IEEE Vehicular Technology Conference 2001 (VTC2001-Fall), vol. 4. 2001, p. $2133-2137$.

[10] NGUYEN, I. S. H. N., ESMAILZADEH, R. A fast scheduling algorithm considering buffer occupancy and channel condition for High Speed Downlink Packet Access. In Proc. $6^{\text {th }}$ International Symposium on Wireless Personal Multimedia Communications (WPMC 2003). 2003, p. $72-76$.

[11] HOLMA, H., TOSKALA, A., RANTA-AHO, K., PIRSKANEN, J. High-speed packet access evolution in $3 \mathrm{GPP}$ release 7. IEEE Communications Magazine, 2007, vol. 45, no. 12, p. 29 - 35 .

[12] MEHLFÜHRER, C., CABAN, S., RUPP, M. Measurement-based performance evaluation of MIMO HSDPA. IEEE Transactions on Vehicular Technology, submitted in Jan. 2010.

[13] MEHLFÜHRER, C., CABAN, S., RUPP, M. Experimental evaluation of adaptive modulation and coding in MIMO WiMAX with limited feedback. EURASIP Journal on Advances in Signal Processing, Special Issue on MIMO Systems with Limited Feedback, 2008, vol. 2008. [Online] Available at: http://publik.tuwien.ac.at/files/pubet_13762.pdf

[14] MEHLFÜHRER, C., CABAN, S., RUPP, M. MIMO HSDPA throughput measurement results in an urban scenario. In Proc. $70^{\text {th }}$ IEEE Vehicular Technology Conference (VTC2009Fall). Anchorage (USA), 2009. [Online] Available at: http://publik.tuwien.ac.at/files/PubDat_176321.pdf

[15] MEHLFÜHRER, C., CABAN, S., GARCÍA-NAYA J. A., RUPP, M. Throughput and capacity of MIMO WiMAX. In Conference Record of the $43^{\text {rd }}$ Asilomar Conference on Signals, Systems and Computers. Pacific Grove (USA), 2009. [Online] Available at: http://publik.tuwien.ac.at/files/PubDat_178050.pdf

[16] ALAMOUTI, S. M. A simple transmit diversity technique for wireless communications. IEEE Journal on Selected Areas in Communications, 1998, vol. 16, no. 8, p. $1451-1458$.
[17] CABAN, S., MEHLFÜHRER, C., LANGWIESER, R., SCHOLTZ, A. L., RUPP, M. Vienna MIMO Testbed. EURASIP Journal on Applied Signal Processing, Special Issue on Implementation Aspects and Testbeds for MIMO Systems, 2006. [Online] Available at: http://publik.tuwien.ac.at/files/pub-et_10929.pdf

[18] CABAN, S., MEHLFÜHRER, C., LECHNER, G., RUPP, M. Testbedding MIMO HSDPA and WiMAX. In Proc. $70^{\text {th }}$ IEEE Vehicular Technology Conference (VTC2009Fall). Anchorage (USA), 2009. [Online] Available at: http://publik.tuwien.ac.at/files/PubDat_176574.pdf

[19] MEHLFÜHRER, C., CABAN, S., RUPP, M. Measurement based evaluation of low complexity receivers for D-TxAA HSDPA. In Proc. $16^{\text {th }}$ European Signal Processing Conference (EUSIPCO 2008). Lausanne (Switzerland), 2008. [Online] Available at: http://publik.tuwien.ac.at/files/PubDat_166132.pdf

[20] MEHLFÜHRER, C., CABAN, S., WRULICH, M., RUPP, M. Joint throughput optimized CQI and precoding weight calculation for MIMO HSDPA. In Conference Record of the $42^{\text {nd }}$ Asilomar Conference on Signals, Systems and Computers. Pacific Grove (USA), 2008, p. 1320 - 1325. [Online] Available at: http://publik.tuwien.ac.at/files/PubDat_167015.pdf

[21] RUPP, M., CABAN, S., MEHLFÜHRER, C. Challenges in building MIMO testbeds. In Proc. 15th European Signal Processing Conference (EUSIPCO 2007). Poznań (Poland), 2007. [Online] Available at: http://publik.tuwien.ac.at/files/PubDat_112138.pdf

[22] RUPP, M., GARCÍA-NAYA, J. A., MEHLFÜHRER, C., CABAN, S., CASTEDO, L. On mutual information and capacity in frequency selective wireless channels. In Proc. IEEE International Conference on Communications (ICC 2010). Cape Town (South Africa), 2010. accepted. [Online] Available at: http://publik.tuwien.ac.at/files/PubDat_184660.pdf 
